\title{
Unified mechanical approach to piezoelectric bender modeling
}

\author{
Robert Dunsch*, Jean-Marc Breguet \\ Ecole Polytechnique Fédérale de Lausanne (EPFL), Laboratoire de systèmes robotiques (LSRO), \\ Station 9, CH-1015 Lausanne, Switzerland
}

Received 28 September 2005; received in revised form 6 June 2006; accepted 13 June 2006

\begin{abstract}
A new analytical modeling approach for piezoelectric bending elements is described. The approach is based on the beam theory under quasi-static equilibrium condition. It uses the theory of superposition of piezoelectric action in the bender and external moments and forces acting on the bender. Due to the differential approach, this model is applicable to any geometrical design for which the beam theory holds. The distinction between the piezoelectric action and the external loads makes the model applicable for any boundary conditions. The bottom-up approach from the electrically induced strain in the piezoelectric part enables the determination of stresses and strains at every point in the bender. The effects on internal strain distribution by the different kinds of actuation where demonstrated in an experiment. The resulting model from this approach will be well suited to design and optimize piezoelectric bending actuators for any purpose. The implied calculation of the strain and stress in the modeling allows the dimensioning of actuators that are equipped with strain gauges as well as the calculation of the electrical properties for capacitive transducers.

(C) 2006 Elsevier B.V. All rights reserved.
\end{abstract}

Keywords: Piezoelectric; Bender; Analytical model; Quasi-static model; Strain; Stress; Cantilever

\section{Introduction}

Piezoelectric benders are used in various applications as AFM cantilevers, positioning devices and force sensors. To achieve the desired performance, the design and optimization process needs numerical and analytical modeling. While the numerical modeling, such as finite element analysis, shows the behavior of a given structure, the analytical model allows the systematic optimization of various parameters. Finite element tools can also handle parametric models and can therefore be used to design and optimize piezoelectric benders. To justify the effort made to establish an analytical model, this model must be far more adaptable to input parameters, like geometry principle and materials, as the corresponding numerical model.

Many approaches to piezoelectric bender modeling have been published. One of the most profound analysis of piezoelectric benders is given by Smits et al. [1,2]. Smits et al. describe the electrical and mechanical behavior of homogeneous and heterogeneous bimorphs and uses a thermodynamical method based on the total energy of the system to calculate the electrical and

\footnotetext{
* Corresponding author. Tel.: +41 21 6935932; fax: +416933866.

E-mail address: robert.dunsch@epfl.ch (R. Dunsch).
}

mechanical parameters. Their model is parametrical in terms of geometry and materials but the boundary condition cannot be changed. It is limited to benders with one free end and clamped on the other side. The input points of forces cannot be chosen. Although this is the most profound analysis, the complexity of the modeling approach does not allow an adaptation to different geometry and boundary conditions.

De Lit et al. [3] use a completely mechanical approach to describe piezoelectric bending actuators. They calculated all external variables, like curvature, tip deflection and tip angle based on the equilibrium of forces and moments. The bender is treated as an Euler-beam for mechanical simplification. This model is also limited in terms of boundary conditions and the influences of external loads onto the electrical parameters are neglected. Weinberg [4] developed a similar model, but includes the influence of external forces onto the charge. Using these parameters he gets a full transducer matrix. This model shows the same limitations concerning geometry and boundary conditions as the ones developed by Smits et al. [1,2] and De Lit et al. [3], but can be applied to benders with several layers. The significance of the work done by Weinberg is that he also treated the problem of multiple neutral planes in the heterogeneous bending elements, but did not mention their importance for the calculation of the real existing stresses and strains in the transducer. Park 


\begin{tabular}{|c|c|}
\hline \multicolumn{2}{|c|}{ Nomenclature } \\
\hline$D$ & electric displacement \\
\hline$d_{31}$ & piezoelectric charge constant \\
\hline$E$ & $\begin{array}{l}\text { Young's-modulus (index s) or electric field (index } \\
3 \ldots \text { ) }\end{array}$ \\
\hline$F$ & force (general) \\
\hline$h_{i}$ & thickness of layers (defined by index) \\
\hline$I$ & moment of inertia \\
\hline$K, N$ & constants for simplification \\
\hline$L$ & length of bender \\
\hline$M$ & moment (general-specified by index) \\
\hline$Q$ & electric charge \\
\hline$S$ & strain tensor \\
\hline$s_{11}^{\mathrm{s}}$ & compliance of support material \\
\hline$s_{11}^{\mathrm{p}}$ & compliance of piezo material \\
\hline$T$ & stress tensor \\
\hline$V$ & voltage \\
\hline$w$ & width of bender \\
\hline$x, y, z$ & space coordinates \\
\hline$z_{0}$ & position of the neutral layer for external loads \\
\hline \multicolumn{2}{|c|}{ Greek letters } \\
\hline$\delta$ & deflection (general) \\
\hline$\varepsilon$ & strain (general) \\
\hline$\varepsilon_{0}$ & strain that defines the bending \\
\hline$\epsilon_{33}$ & $\begin{array}{l}\text { dielectric constant of the piezo material (in fact } \\
\epsilon_{33}^{T} \text { ) }\end{array}$ \\
\hline$\gamma$ & $\begin{array}{l}\text { position of the neutral layer for piezoelectric bend- } \\
\text { ing }\end{array}$ \\
\hline$\sigma$ & stress (general) \\
\hline$\varrho$ & radius of curvature \\
\hline
\end{tabular}

and Moon [5] established a model addressing the problem of various boundary conditions. This article [5] shows an approach to handle the influence of the boundaries onto the external loads but stays limited to a fixed set of boundaries.

All the articles mentioned have some similarities. They are based on the static equilibrium of forces and moments. In every case, the assumption is made that a superposition of piezoelectric action and external loads in the model is possible. These assumptions are also made by Wiesendanger [6], but he uses a total different approach. In his work, the influence of external mechanical loads onto electrical and mechanical behavior is neglected, but the calculation of strains and stresses is the basis of the determination of the actuators deflection. Although this approach was used by Wiesendanger in a very limited way, it does not only allow the calculation of any mechanical parameter but also the determination of the real strains and stresses in the beam. These values are of vital importance for applications with integrated strain gauges and the calculation of internal electromechanical effects.

The modeling approach presented in this article will use this background and superpose the effects of piezoelectric action and

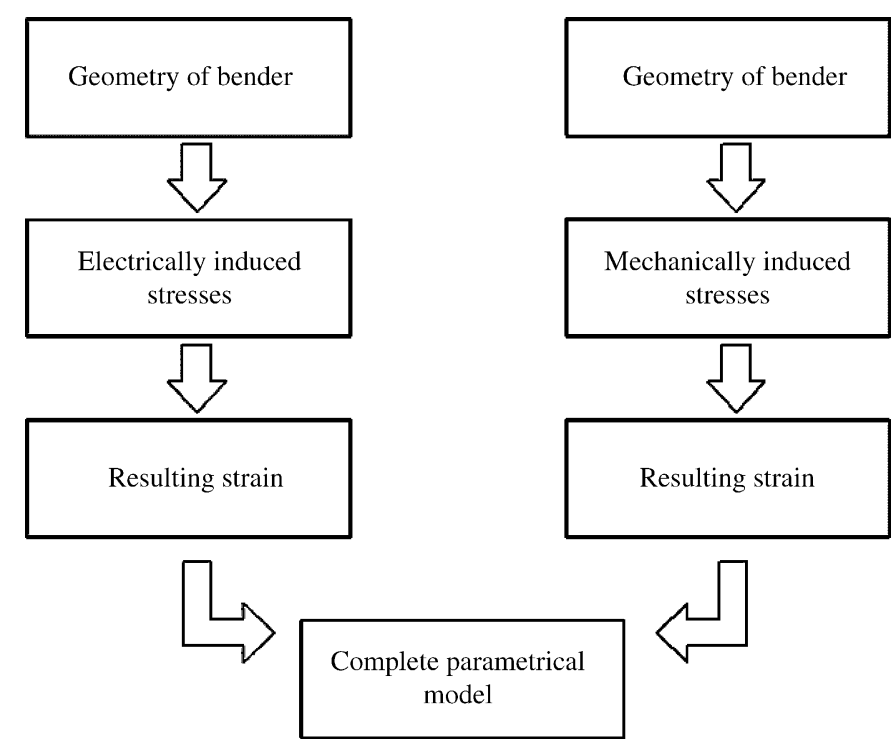

Fig. 1. Schematic of the modeling approach.

external loads in a complete model. The approach is shown in Fig. 1. This paper describes a new modeling approach that leads to a static model for all possible piezoelectric bender actuators under any lateral loads. It will be based on simple mechanical theories that allows an easy extension according to the complexity of the modeled system.

\section{Derivation of equations}

The equations describing the behavior of the bender are based on the strain in the material and separated into two parts: the equations of the piezoelectric action and the equations of the bender under external mechanical load.

For piezoelectric benders, three different configurations are of interest (see Fig. 2): the triple-layer bender (a), consisting of two piezoelectric layers acting in opposite manner and a mechanically relevant passive layer in between; the bimorph (b), that has the same configuration but without the intermediate layer; the heterogeneous bimorph (c-also called monomorph or unimorph), that consists of one piezoelectric layer and one passive. The three cases are given in Fig. 2.

The model is established for triple-layer bender configuration, the others can be derived by setting the thicknesses of the non-existing layers to zero. For the modeling process, some general assumptions have to be made:

(1) For the benders, the Euler-Bernoulli-beam-theory holds.

(2) There is no slip between the layers (the strain is continuous over the thickness).

(3) The beam is in a static equilibrium: $\Sigma F=0 ; \Sigma M=0$.

From the general equations of piezoelectricity, the constituent equations of the bimorphic bender can be derived [1]. In a bimorph or triple-layer, bender the equation for the lower and upper piezoelectric layer have an opposite sign, for a monomorph the upper layer will be considered piezoelectric and the lower passive. The superscripts u (upper) and 1 (lower) 

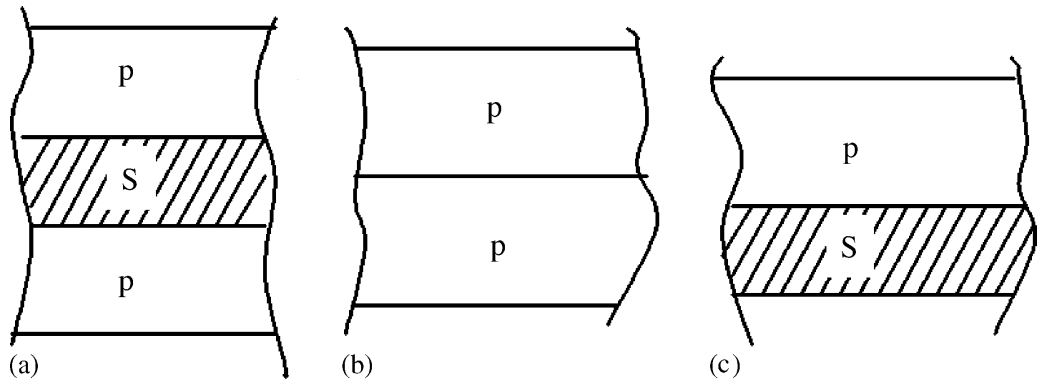

(b)

(c)

Fig. 2. Three possible bender configurations (p, piezo and s, passive layer): (a) triple-layer bender, (b) bimorph and (c) monomorph.

indicate the position of the layer. The subscripts 1-3 represent the coordinates $x-z$, respectively, according to Fig. 3 .

$$
\begin{aligned}
& S_{1}^{\mathrm{u}}=s_{11}^{E} T_{1}^{\mathrm{u}}-d_{31} E_{3}^{\mathrm{u}} \\
& D_{3}^{\mathrm{u}}=-d_{31} T_{1}^{\mathrm{u}}+\epsilon_{33} E_{3}^{\mathrm{u}} \\
& S_{1}^{1}=s_{11}^{E} T_{1}^{1}+d_{31} E_{3}^{1} \\
& D_{3}^{1}=d_{31} T_{1}^{1}+\epsilon_{33} E_{3}^{1}
\end{aligned}
$$

For most technically used piezoelectric materials holds $d_{31}<$ 0 that defines the sign in the right-hand term of Eqs. (1) and (3). The parameter $s_{11}^{E}$ is the compliance of the piezoelectric material [7]. For non-piezoelectric materials usually the Young's modulus $E$ is given. To avoid confusion between $E_{3}$, the electric field and $E_{\mathrm{s}}$, the Young's modulus of the support layer and to have a consistent outline of the formulas the compliance $s_{11}^{\mathrm{s}}$ will be used instead. This compliance is defined:

$s_{11}^{\mathrm{s}}=\frac{1}{E_{\mathrm{s}}}$.

For the whole model, the correct use of the sign is of great importance. The positive directions of all parameters are given in Fig. 3: in this article, the beams will be considered prismatic with a rectangular section of the width $w$, that will be constant over the length and equal for all layers.

\subsection{Piezoelectric bending}

The effects of piezoelectric bending and bending due to external loads will be considered separately. The piezoelectric bending results from the compression or extension in the piezoelectric material enforced by the electric field. This is the mechanical base of all piezoelectric actuation. As stated in the beginning of Section 2, the calculation will be based on a static equilibrium.

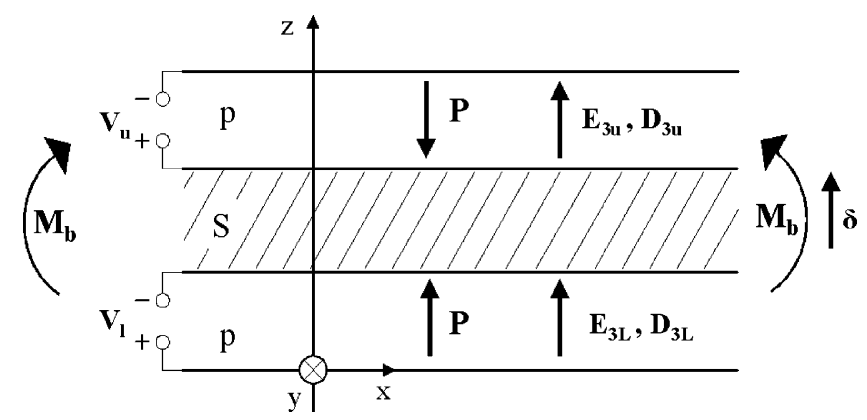

Fig. 3. Sign convention for the bender model (p, piezo and s, passive layer).
A detailed description of the basics of piezoelectric bending can be found in ref. [1]. The actuation in the bender results from the strain $\varepsilon_{\mathrm{u}}$ induced by the electric field $E_{3}$ and the piezoelectric material property defined by the constant $d_{31}$ :

$\varepsilon_{\mathrm{u}}=-d_{31} E_{3}$.

The Hooke's law of stress and strain is:

$\sigma=\varepsilon E=\frac{\varepsilon}{s_{11}}$.

The acting strains and stresses in a symmetric trimorph bender are depicted in Fig. 4. In this case, the neutral plane $N_{0}$ is in the center of the beam. The strain in the piezoelectric parts, induced by the electric field $\varepsilon_{\mathrm{u}}$, is positive in the upper layer and negative in the lower layer. The desire to elongate the upper piezo layer and contract the lower piezo layer, respectively, causes the stress in the upper layer $\sigma_{\mathrm{pu}}$ to be negative and the stress in the lower piezo layer $\sigma_{\mathrm{pl}}$ to be positive.

Two parameters define the bending of the beam: The internal strain $\varepsilon_{0} z$ and the position of the neutral plane. They can be calculated by solving the equations of the static equilibrium $\Sigma F=0 ; \Sigma M=0$. With the stresses $\sigma_{\mathrm{pu}}, \sigma_{\mathrm{pl}}$ and $\sigma_{\mathrm{s}}$ we can calculate the force and the moment acting in the bender by inte-

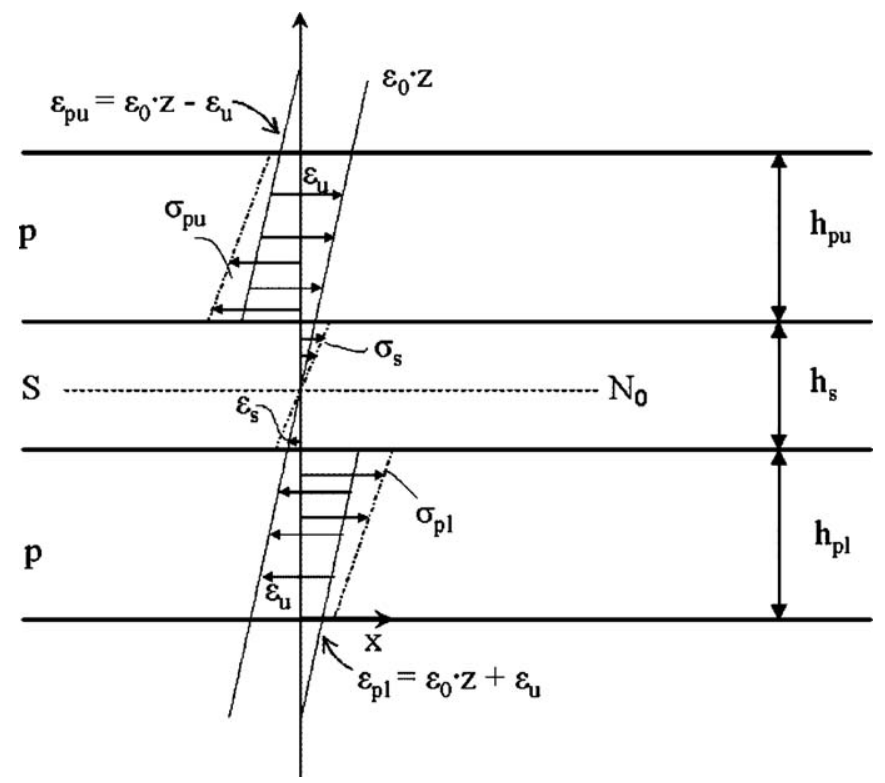

Fig. 4. Acting strains and stresses in the bender. 


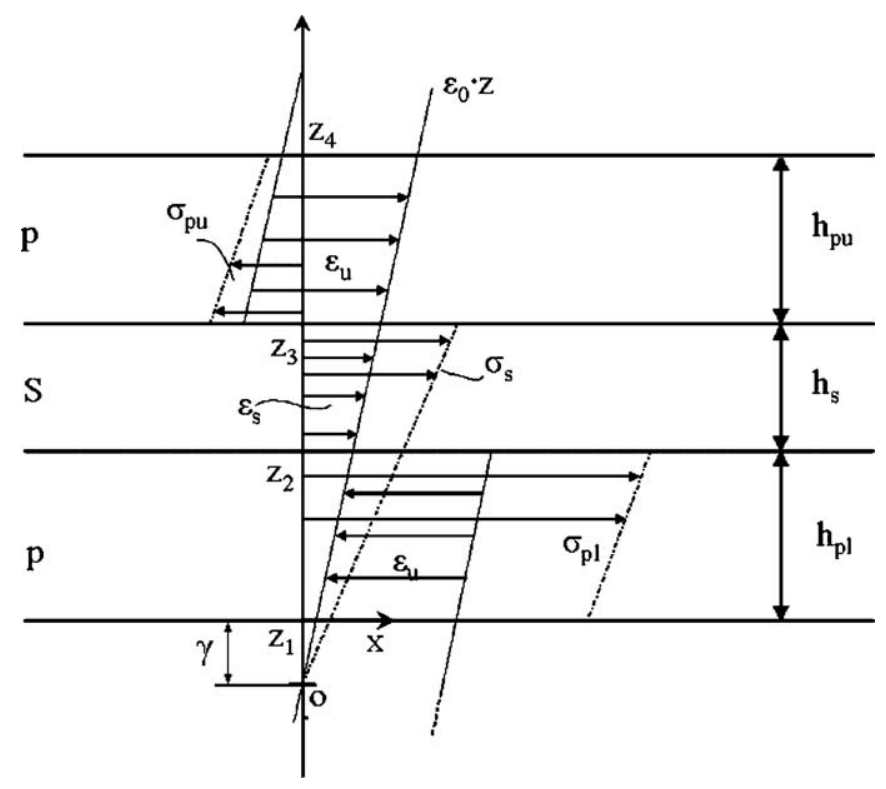

Fig. 5. Strains and stresses in the bender (while the point of zero strains and stresses $o$ is moved virtually outside).

grating the stress $\sigma$ over the cross-sectional area $A$.

$F=\iint_{(A)} \sigma \mathrm{d} y \mathrm{~d} z$

$M=\iint_{(A)} \sigma z \mathrm{~d} y \mathrm{~d} z$

The cross-sectional area $A$ is determined by the width of the bender $w$ and the heights $h_{\mathrm{s}}$ and $h_{\mathrm{pu}}, h_{\mathrm{pl}}$, respectively. For the calculation, we move the neutral plane virtually outside of the beam to the position $o$. This operation has the advantage, that the position of the neutral plane appears in the limits of the integration. The global origin of the coordinate system stays in place according to Fig. 3. This virtual operation is shown in Fig. 5.

The distance of the neutral plane from the origin of the global coordinate system (i.e. the bottom of the bender) is called $\gamma$. The limits of the integration, which are the edges of the layers, are with respect to the position of the neutral plane:

$z_{1}=\gamma ; \quad z_{2}=z_{1}+h_{\mathrm{pl}} ; \quad z_{3}=z_{2}+h_{\mathrm{s}} ; \quad z_{4}=z_{3}+h_{\mathrm{pu}}$.

The stresses in the bender are according to Fig. 5:

$\sigma_{\mathrm{pu}}=\frac{1}{s_{11}^{\mathrm{p}}} w\left(\varepsilon_{0} z-d_{31} E_{3}^{\mathrm{u}}\right)$,

$\sigma_{\mathrm{pl}}=\frac{1}{s_{11}^{\mathrm{p}}} w\left(\varepsilon_{0} z+d_{31} E_{3}^{1}\right)$,

$\sigma_{\mathrm{s}}=\frac{1}{s_{11}^{\mathrm{s}}} w\left(\varepsilon_{0} z\right)$.

The electric field $E$ has always the same direction, but since the polarization in the two piezoelectric layers is opposite, its effect is positive for the lower layer and negative for the upper layer. With Eqs. (8) and (9), we can now calculate the resulting force and moment (with $w=$ const. the integration is about $z$ only).

$$
\begin{aligned}
F= & \int_{z_{1}}^{z_{2}}\left(\frac{1}{s_{11}^{\mathrm{p}}} w\left(\varepsilon_{0} z-d_{31} E_{3}^{1}\right)\right) \mathrm{d} z \\
& +\int_{z_{2}}^{z_{3}}\left(\frac{1}{s_{11}^{\mathrm{s}}} w\left(\varepsilon_{0} z\right)\right) \mathrm{d} z \\
& +\int_{z_{3}}^{z_{4}}\left(\frac{1}{s_{11}^{\mathrm{p}}} w\left(\varepsilon_{0} z+d_{31} E_{3}^{\mathrm{u}}\right)\right) \mathrm{d} z \\
M= & \int_{z_{1}}^{z_{2}}\left(\frac{1}{s_{11}^{\mathrm{p}}} w\left(\varepsilon_{0} z-d_{31} E_{3}^{1}\right)\right) z \mathrm{~d} z \\
& +\int_{z_{2}}^{z_{3}}\left(\frac{1}{s_{11}^{\mathrm{s}}} w\left(\varepsilon_{0} z\right)\right) z \mathrm{~d} z \\
& +\int_{z_{3}}^{z_{4}}\left(\frac{1}{s_{11}^{\mathrm{p}}} w\left(\varepsilon_{0} z+d_{31} E_{3}^{\mathrm{u}}\right)\right) z \mathrm{~d} z
\end{aligned}
$$

With the equilibrium condition ( $\Sigma F=0 ; \Sigma M=0$ for no external loads) these equations can be solved for $\gamma$ and $\varepsilon_{0}$. For the complete triple-layer bender follows:

$$
\begin{aligned}
\varepsilon_{0}= & -\frac{6 d_{31} s_{11}^{\mathrm{s}} h_{\mathrm{s}}\left(E_{3}^{1} h_{\mathrm{pl}}\left(h_{\mathrm{pl}}+h_{\mathrm{s}}\right)+E_{3}^{\mathrm{u}} h_{\mathrm{pu}}\left(h_{\mathrm{pu}}+h_{\mathrm{s}}\right)\right) s_{11}^{\mathrm{p}}}{K} \\
& -\frac{6 d_{31} s_{11}^{\mathrm{s}}\left(E_{3}^{1}+E_{3}^{\mathrm{u}}\right) h_{\mathrm{pl}} h_{\mathrm{pu}}\left(h_{\mathrm{pl}}+h_{\mathrm{pu}}+2 h_{\mathrm{s}}\right) s_{11}^{\mathrm{s}}}{K}
\end{aligned}
$$

with

$$
\begin{aligned}
K= & h_{\mathrm{s}}^{4} s_{11}^{\mathrm{p} 2}+2 h_{\mathrm{s}}\left(2\left(h_{\mathrm{pl}}^{3}+h_{\mathrm{pu}}^{3}\right)+3\left(h_{\mathrm{pl}}^{2}+h_{\mathrm{pu}}^{2}\right) h_{\mathrm{s}}\right. \\
& \left.+2\left(h_{\mathrm{pl}}+h_{\mathrm{pu}}\right) h_{\mathrm{s}}^{2}\right) s_{11}^{\mathrm{p}} s_{11}^{\mathrm{s}}+\left(\left(h_{\mathrm{pl}}+h_{\mathrm{pu}}\right)^{4}\right. \\
& \left.+12 h_{\mathrm{pl}} h_{\mathrm{pu}}\left(h_{\mathrm{pl}}+h_{\mathrm{pu}}\right) h_{\mathrm{s}}+12 h_{\mathrm{pl}} h_{\mathrm{pu}} h_{\mathrm{s}}^{2}\right) s_{11}^{\mathrm{s} 2}
\end{aligned}
$$

and

$$
\begin{aligned}
& \gamma=-\frac{\left(E_{3}^{1} h_{\mathrm{pl}}\left(6 h_{\mathrm{pl}}^{2}+9 h_{\mathrm{pl}} h_{\mathrm{s}}+4 h_{\mathrm{s}}^{2}\right)\right) s_{11}^{\mathrm{p}} h_{\mathrm{s}}}{N} \\
& -\frac{\left(E_{3}^{\mathrm{u}} h_{\mathrm{pu}}\left(6 h_{\mathrm{pl}}\left(h_{\mathrm{pu}}+h_{\mathrm{s}}\right)+h s\left(3 h_{\mathrm{pu}}+2 h_{\mathrm{s}}\right)\right)\right) s_{11}^{\mathrm{s}} h_{\mathrm{s}}}{N} \\
& \left(h_{\mathrm{pl}}+h_{\mathrm{pu}}\right)^{2}\left(E_{3}^{\mathrm{u}} h_{\mathrm{pu}}\left(2 h_{\mathrm{pl}}-h_{\mathrm{pu}}\right)\right. \\
& -\frac{\left.+E_{3}^{1} h_{\mathrm{pl}}\left(h_{\mathrm{pl}}+4 h_{\mathrm{pu}}\right)\right) s_{11}^{\mathrm{s}}}{N} \\
& \left(6 h_{\mathrm{pl}} h_{\mathrm{pu}}\left(3 E_{3}^{1} h_{\mathrm{pl}}+E_{3}^{\mathrm{u}} h_{\mathrm{pl}}+2 E_{3}^{1} h_{\mathrm{pu}}\right) h_{\mathrm{s}}\right. \\
& -\frac{\left.+12 E_{3}^{1} h_{\mathrm{pl}} h_{\mathrm{pu}} h_{\mathrm{s}}^{2}\right) s_{11}^{\mathrm{s}}}{N}
\end{aligned}
$$

with

$$
\begin{aligned}
N= & 6 h_{\mathrm{s}}\left(E_{3}^{1} h_{\mathrm{pl}}\left(h_{\mathrm{pl}}+h_{\mathrm{s}}\right)+E_{3}^{\mathrm{u}} h_{\mathrm{pu}}\left(h_{\mathrm{pu}}+h_{\mathrm{s}}\right)\right) s_{11}^{\mathrm{p}} \\
& +6\left(E_{3}^{1}+E_{3}^{\mathrm{u}}\right) h_{\mathrm{pl}} h_{\mathrm{pu}}\left(h_{\mathrm{pl}}+h_{\mathrm{pu}}+2 h_{\mathrm{s}}\right) s_{11}^{\mathrm{s}} .
\end{aligned}
$$

The values for $\gamma$ are negative, since the point $o$ in Fig. 5 was chosen outside of the beam, and the origin of the global coordinate system is at the bottom of the whole bender (see Fig. 3). 
The point of zero stress and strain $\gamma$ is the neutral plane for piezoelectric bending, $\varepsilon_{0}$ stands for the strain slope inside the bender. The exact strain in is therefore:

$\varepsilon(z)=\varepsilon_{0}(z+\gamma)$.

The strain slope $\varepsilon_{0}$ is also the reciprocal value of the benders radius of curvature:

$\varepsilon_{0}=\frac{1}{\varrho}$.

According to the beam theory, the deflection $\delta(x)$ of the bender can be calculated by the double integration with respect to $x$.

$\delta^{\prime}(x)=\int \varepsilon_{0} \mathrm{~d} x, \quad \delta(x)=\int \delta^{\prime}(x) \mathrm{d} x$

Taken a simple symmetric bimorph of the length $L$ and the layer thickness $h$, clamped on one side, the deflection of the free end, calculated with Eq. (21), becomes (with $E_{3}=\frac{V}{2 h}$ ):

$\delta(L)=\frac{3 d_{31} L^{2}}{8 h^{2}} V$

This is exactly the result Smits et al. [1] achieves for this configuration in his paper.

\subsection{Mechanical properties}

Besides the piezoelectric actuation, the bender is also subject to external moments and forces. For this section, the bender behaves as a passive composite beam. All occurring external loads will be merged to one parameter $M_{\mathrm{b}}(x)$, that may vary over the beams length. The calculation of the bending moment is done according to the basics of mechanics [8], and will not be treated here. Other important mechanical properties are the moment of inertia $I$ and the mechanical neutral plane $z_{0}$. This neutral plane $z_{0}$ is not equal to the neutral plane of piezoelectric bending $\gamma$, which is explained in Section 2.4.

The mechanical neutral plane is calculated from the force equilibrium. With Eq. (8), we can calculate the force from the stress in the beam. The stress resulting from external loads in the beam is:

$\sigma=\frac{\varepsilon_{M}}{s_{11}}\left(z-z_{0}\right)$,

while $\varepsilon_{M}$ is the mechanically induced strain slope according to $\varepsilon_{0}$, defined in Eq. (30). Therefore, the force $F$ is (for constant width $w$ ):

$F=\int_{z} \frac{\varepsilon_{M}}{s_{11}} w\left(z-z_{0}\right) \mathrm{d} z$

For the whole beam, the force equilibrium is:

$$
\begin{aligned}
F= & \int_{0}^{h_{\mathrm{pl}}} \frac{\varepsilon_{M}}{s_{11}^{\mathrm{p}}} w\left(z-z_{0}\right) \mathrm{d} z+\int_{h_{\mathrm{pl}}}^{h_{\mathrm{pl}}+h_{\mathrm{s}}} \frac{\varepsilon_{M}}{s_{11}^{\mathrm{s}}} w\left(z-z_{0}\right) \mathrm{d} z \\
& +\int_{h_{\mathrm{pl}}+h_{\mathrm{s}}}^{h_{\mathrm{pl}}+h_{\mathrm{s}}+h_{\mathrm{pu}}} \frac{\varepsilon_{M}}{s_{11}^{\mathrm{p}}} w\left(z-z_{0}\right) \mathrm{d} z=0
\end{aligned}
$$

For $z_{0}$ one obtains:

$$
\begin{gathered}
h_{\mathrm{s}}^{2} s_{11}^{\mathrm{p}}+h_{\mathrm{pl}}^{2} s_{11}^{\mathrm{s}}+h_{\mathrm{pu}}^{2} s_{11}^{\mathrm{s}}+2 h_{\mathrm{pu}} h_{\mathrm{s}} s_{11}^{\mathrm{s}} \\
z_{0}=\frac{+2 h_{\mathrm{pl}}\left(h_{\mathrm{s}} s_{11}^{\mathrm{p}}+h_{\mathrm{pu}} s_{11}^{\mathrm{s}}\right)}{2\left(h_{\mathrm{s}} s_{11}^{\mathrm{p}}+\left(h_{\mathrm{pl}}+h_{\mathrm{pu}}\right) s_{11}^{\mathrm{s}}\right)} .
\end{gathered}
$$

The moment of inertia $I$ is calculated according to the definition:

$I=\int_{(A)} z^{2} \mathrm{~d} A$

where $A$ is the cross-sectional area of the bender. The moment of inertia is independent of the Young's modulus for beams of one material. The bender is, however, except for the bimorph, a composite structure. For the correct moment of inertia, the cross-sections will be weighted according to their Young's modulus. The reference modulus will be here $s_{11}^{\mathrm{s}}$. The moment of inertia for the total bender calculates according to this:

$$
\begin{aligned}
I= & w \frac{s_{11}^{\mathrm{s}}}{s_{11}^{\mathrm{p}}} \int_{-z_{0}}^{h_{\mathrm{pl}}-z_{0}} z^{2} \mathrm{~d} z+w \int_{h_{\mathrm{pl}}-z_{0}}^{h_{\mathrm{pl}}+h_{\mathrm{s}}-z_{0}} z^{2} \mathrm{~d} z \\
& +w \frac{s_{11}^{\mathrm{s}}}{s_{11}^{\mathrm{p}}} \int_{h_{\mathrm{pl}}+h_{\mathrm{s}}-z_{0}}^{h_{\mathrm{pl}}+h_{\mathrm{s}}+h_{\mathrm{pu}}-z_{0}} z^{2} \mathrm{~d} z .
\end{aligned}
$$

Simplification gives:

$I=\frac{K w}{12 s_{11}^{\mathrm{p}}\left(h_{\mathrm{s}} s_{11}^{\mathrm{p}}+\left(h_{\mathrm{pl}}+h_{\mathrm{pu}}\right) s_{11}^{\mathrm{s}}\right)}$,

with $K$ from Eq. (16). With these parameters, the mechanical behavior of the bender can be calculated. The real strain in the bender due to external loads is:

$\varepsilon_{M}=\frac{s_{11}^{\mathrm{s}}}{I} M_{\mathrm{b}}(x)\left(z-z_{0}\right)$.

\subsection{Superposition of mechanical properties and piezoelectric actuation}

As mentioned before, the final physical behavior of the bender is a superposition of the piezoelectric actuation and the passive bending under external loads. The total strain in the bender is:

$\varepsilon_{\mathrm{tot}}(z, x)=\varepsilon_{0}(z+\gamma)+\frac{s_{11}^{\mathrm{s}}}{I} M_{\mathrm{b}}(x)\left(z-z_{0}\right)$.

For the calculation of the curvature and the deflection of the beam, the zero-positions of the strain are not necessary.

$\frac{1}{\varrho}=\varepsilon_{\mathrm{tot}}=\varepsilon_{0}+\frac{s_{11}^{\mathrm{s}}}{I} M_{\mathrm{b}}(x)$

With $\varepsilon_{\text {tot }}$ the curvature and related properties like the deflection can be calculated according to Eqs. (20) and (21).

\subsection{Origin and relevance of multiple neutral planes}

As stated in Sections 2.1 and 2.2, the points of zero stresses and strains (i.e. the neutral plane) for piezoelectric actuation and external loading do not coincide necessarily. For 


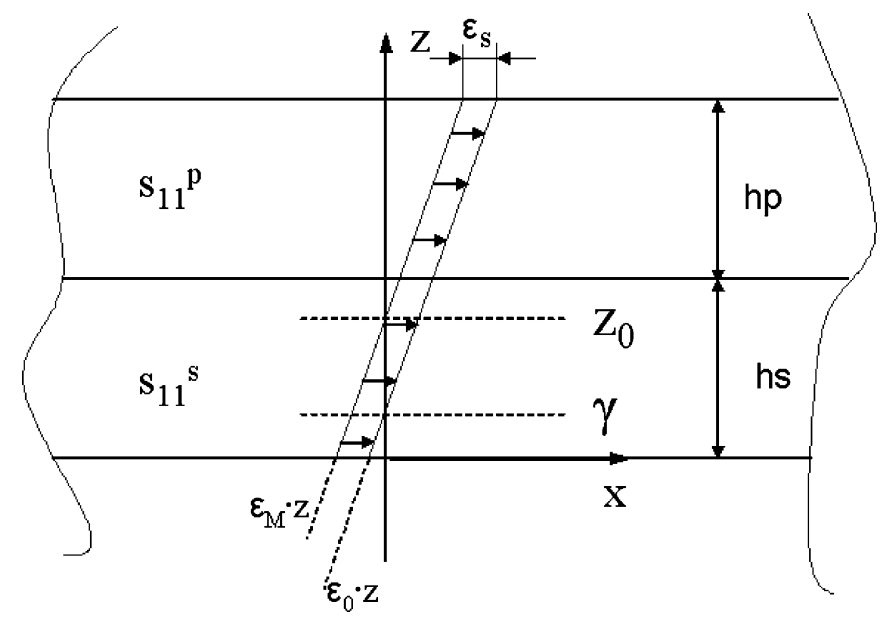

Fig. 6. Strains in a heterogeneous bimorph for piezoelectric bending and bending due to an external moment.

benders of symmetric geometry, also taking into account the material properties, only one neutral plane exists. For nonsymmetric benders, as the heterogeneous bimorphs this is not the case. The values for $\gamma$ and $z_{0}$ are different (see Eqs. (17) and (26)).

The reason of this effect can be found in the main difference between piezoelectric bending and bending under external load. While the external loads considered in this article consists only of lateral forces and moments, the piezoelectric bending is caused by a longitudinal force (i.e. the elongation/contraction of the piezoelectric part).

For a heterogeneous bimorph (monomorph) according to Fig. 2(c), with the piezo layer thickness $h_{\mathrm{p}}$, the passive layer thickness $h_{\mathrm{s}}$ and the associated compliances $s_{11}^{\mathrm{p}}$ and $s_{11}^{\mathrm{s}}$, the Eqs. (17) and (26) simplify to:

$\gamma=-\frac{3 h_{\mathrm{p}} h_{\mathrm{s}}^{2} s_{11}^{\mathrm{p}}+2 h_{\mathrm{s}}^{3} s_{11}^{\mathrm{p}}-h_{\mathrm{p}}^{3} s_{11}^{\mathrm{s}}}{6 h_{\mathrm{s}} s_{11}^{\mathrm{p}}\left(h_{\mathrm{p}}+h_{\mathrm{s}}\right)}$

$z_{0}=\frac{h_{\mathrm{s}}^{2} s_{11}^{\mathrm{p}}+h_{\mathrm{p}}^{2} s_{11}^{\mathrm{s}}+2 h_{\mathrm{p}} h_{\mathrm{s}} s_{11}^{\mathrm{s}}}{2\left(h_{\mathrm{s}} s_{11}^{\mathrm{p}}+h_{\mathrm{p}} s_{11}^{\mathrm{s}}\right)}$.

It can be seen that they are not equal. The strain inside a beam of this geometry under piezoelectric bending and bending under an external moment is shown in Fig. 6. The external moment is chosen in a way that the radius of curvature $\varrho$ is the same as for the piezoelectric actuation (i.e. $\varepsilon_{0}=\varepsilon_{M}$ ).

The schematic shows that there is a constant difference $\varepsilon_{\mathrm{S}}$ between the strains due to piezoelectric bending and due to the external moment:

$\varepsilon_{0}(z+\gamma)=\varepsilon_{M}\left(z-z_{0}\right)+\varepsilon_{\mathrm{s}}$

If the piezoelectric bending is considered a combination of a longitudinal force and a bending moment, the strain is also a combination of strains caused by these two effects. Hence, the strain offset $\varepsilon_{\mathrm{s}}$ is the part caused by the longitudinal force.

To calculate this offset a thought experiment is conducted. A heterogeneous bimorph of under piezoelectric actuation is

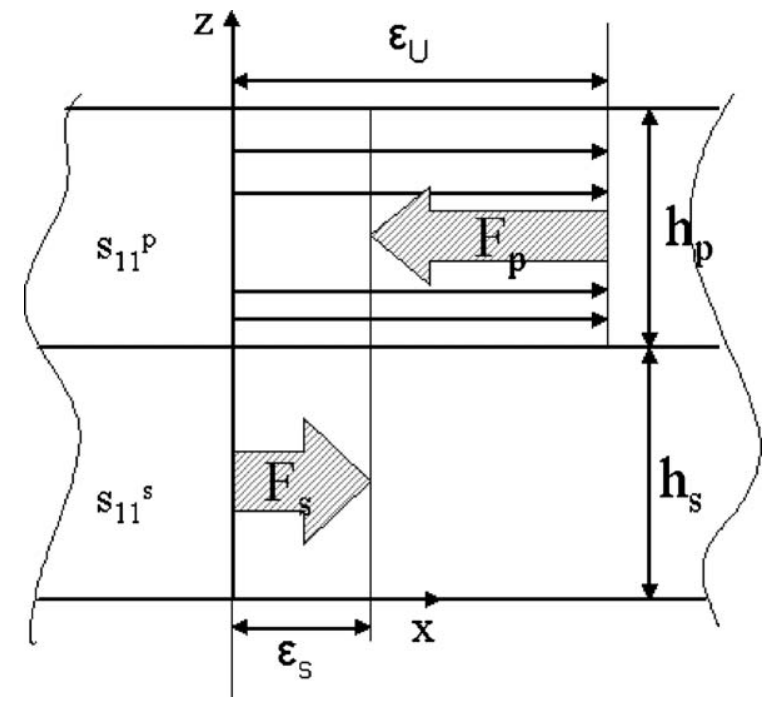

Fig. 7. Constant strains in a heterogeneous bimorph that is forced in a straight shape (no bending).

hindered to bend. In the totally straight beam all strains are uniformly distributed over the thickness of the layer. The effect is shown in Fig. 7.

According to this assumption, we can calculate the strain offset $\varepsilon_{\mathrm{S}}$ from the static force equilibrium. The calculation includes only length-forces (absolutely no bending). The elongation of the piezo part is defined in Eq. (6). Now the support layer is stretched and the piezo layer is shortened, resulting in uniformely distributed strains. The strain in the piezo layer is called $\varepsilon_{\mathrm{p}}$.

$\varepsilon_{\mathrm{U}}=\varepsilon_{\mathrm{s}}+\varepsilon_{\mathrm{p}}$

The forces $F_{\mathrm{p}}$ and $F_{\mathrm{s}}$ are, according to Eqs. (7) and (8):

$F_{\mathrm{p}}=\frac{1}{s_{11}^{\mathrm{p}}}\left(\varepsilon_{\mathrm{p}} w h_{\mathrm{p}}\right)$

$F_{\mathrm{s}}=\frac{1}{s_{11}^{\mathrm{s}}}\left(\varepsilon_{\mathrm{s}} w h_{\mathrm{s}}\right)$

With the static equilibrium of forces $\left(\sum F=0\right)$, we can express $\varepsilon_{\mathrm{p}}$ as a function of $\varepsilon_{\mathrm{s}}$ :

$\varepsilon_{\mathrm{p}}=\frac{s_{11}^{\mathrm{p}} h_{\mathrm{s}}}{s_{11}^{\mathrm{s}} h_{\mathrm{p}}} \varepsilon_{\mathrm{s}}$

Input this into Eq. (36), we find:

$\varepsilon_{\mathrm{s}}=\varepsilon_{\mathrm{u}} \frac{s_{11}^{\mathrm{s}} h_{\mathrm{p}}}{s_{11}^{\mathrm{s}} h_{\mathrm{p}}+s_{11}^{\mathrm{p}} h_{\mathrm{s}}}$

Now, we create an auxiliary strain variable $\varepsilon_{M}^{*}$ that is numerically equal to $\varepsilon_{M}$ :

$\varepsilon_{M}^{*}=\varepsilon_{0}(z-\gamma)+\varepsilon_{\mathrm{s}}$ 


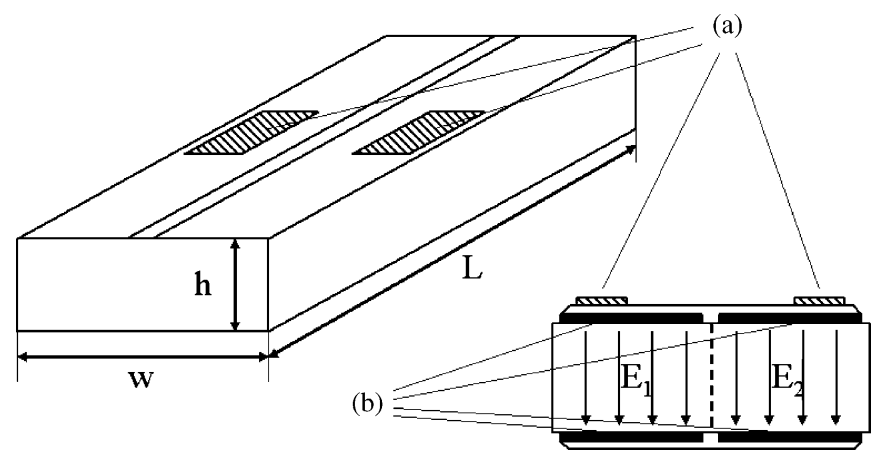

Fig. 8. Planar bimorph for experimentation, with: (a) strain gauges and (b) electrodes.

with $\varepsilon_{0}$ from Eq. (15), simplified for the used geometry. Set $\varepsilon_{M}^{*}$ to zero and solved for $z$ yields:

$z\left(\varepsilon_{M}^{*}=0\right)=\frac{h_{\mathrm{s}}^{2} s_{11}^{\mathrm{p}}+h_{\mathrm{p}}^{2} s_{11}^{\mathrm{s}}+2 h_{\mathrm{p}} h_{\mathrm{s}} s_{11}^{\mathrm{s}}}{2\left(h_{\mathrm{s}} s_{11}^{\mathrm{p}}+h_{\mathrm{p}} s_{11}^{\mathrm{s}}\right)}$,

which is exactly the same result as Eq. (34).

The effect of the multiple neutral planes was also measured experimentally to show the strain behavior a planar bimorph was equipped with two strain gauges to measure the strain on the surface (Fig. 8), as described in [10].

The electrodes of the beam are not interconnected, thus the electric fields $E_{1}$ and $E_{2}$ can be controlled independently. The beam has the following properties:

$w=2.85 \mathrm{~mm}, \quad h=0.5 \mathrm{~mm}, \quad L=27 \mathrm{~mm}$.

If only one pair of electrodes is carrying a voltage $\left(E_{1} \neq\right.$ $0 ; E_{2}=0$ or $E_{2} \neq 0 ; E_{1}=0$ ), the beam is behaving like a heterogeneous bimorph. Mechanically, however, the beam is absolutely symmetric thus the neutral plane for external loads is in the center. The measurement results are shown in Fig. 9. The experiments A and B were the actuation of either the left or the right pair of electrodes. The diagrams show the strain distribution according to Eq. (19) for several electric fields. The graphs show that the strain is zero for one single point under different electric field strengths. The difference from the calculated neutral plane can be explained by fabrication tolerances of the specimen.

The results of the experiments, the measured positions of the neutral planes, are shown in the following table:

\begin{tabular}{ll}
\hline $\begin{array}{l}\text { Measured position } \\
\text { (experiment A) }(\mathrm{mm})\end{array}$ & $\begin{array}{l}\text { Measured position } \\
(\text { experiment B) }(\mathrm{mm})\end{array}$ \\
\hline 0.420 & 2.554 \\
0.419 & 2.553 \\
0.426 & 2.551 \\
0.425 & 2.539 \\
\hline
\end{tabular}

The values from the four experiments (four different electric field strengths) show a significant accuracy in the range of some micrometers. The average values of the experimental results are listed in the following table and compared to the analytically calculated values.

\begin{tabular}{ll}
\hline Measured position (average) $(\mathrm{mm})$ & Calculated position $(\mathrm{mm})$ \\
\hline 0.423 & 0.441 \\
2.549 & 2.41 \\
\hline
\end{tabular}

The calculation above and the experiments showed the derivation of the difference of neutral planes from the geometry of the bender. The influence of the neutral plane position is very important. In applications, where strain gauges are involved, the knowledge about the real strain distribution is of great importance for design and optimization. The other important point is the influence on the electrical parameters as described in the following section. The electrical parameters in piezoelectric el-
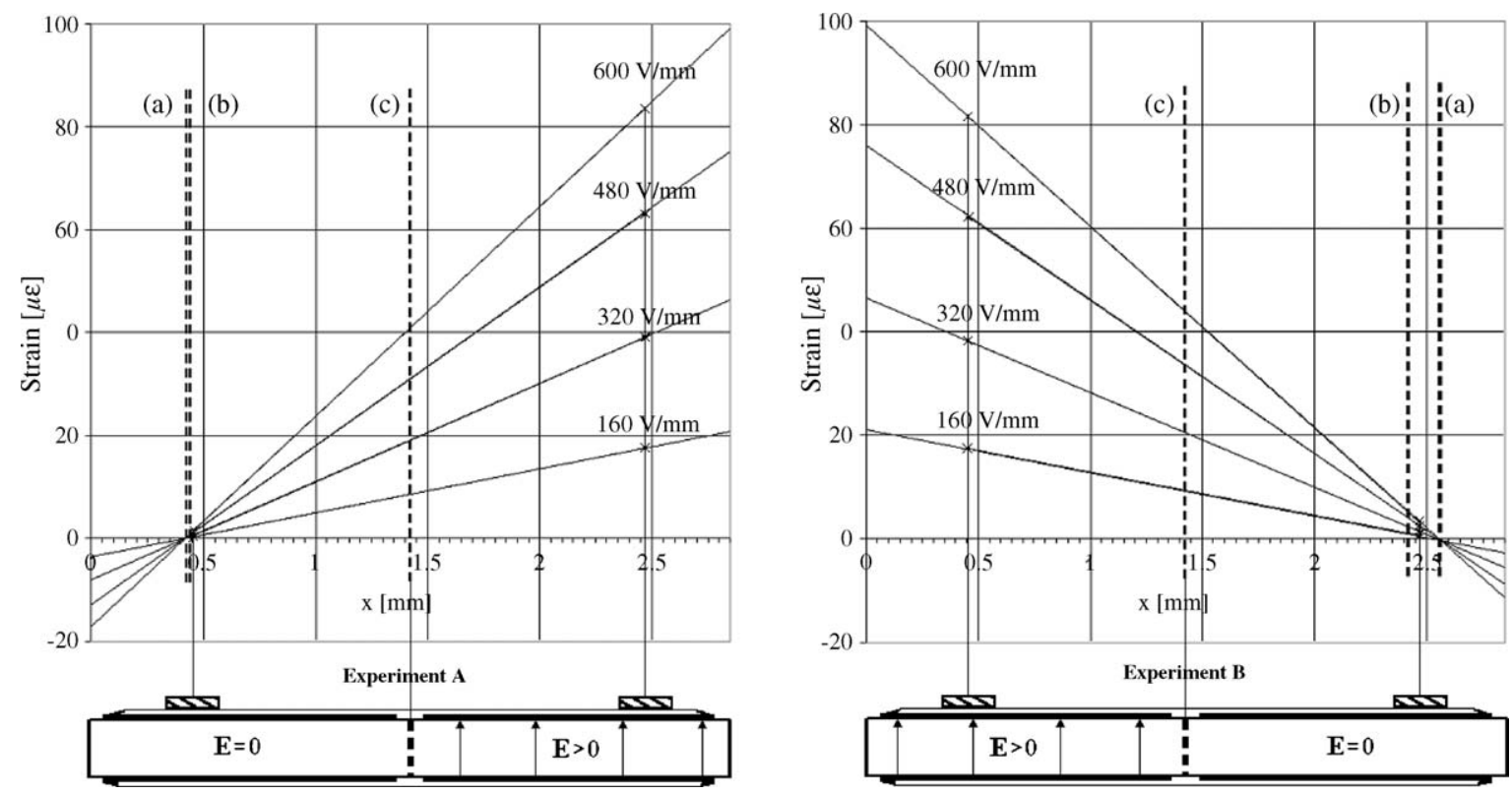

Fig. 9. Results of the experiment, with: (a) measured neutral plane, (b) calculated neutral plane and (c) neutral plane for external loads (center). 
ements are determined mainly by the internal stress distribution which directly depends on the position of the neutral plane. The influence of the exact position of the neutral plane on the calculation of electrical parameters of piezoelectric elements can be seen in Fig. 6.

\subsection{Electrical properties}

Piezoelectric actuators or sensors are electromechanically coupled systems. Any change of electrical parameters will change the mechanical side and vice versa. For sensor applications, the mechanical effect on electrical parameters is of great importance (direct piezoelectric effect). Simple passive sensors output a voltage $V$ depending on the mechanical strain or stress. In a coupled system, however, the voltage (electrical field $E$ ) is an input parameter, enforced by the electrical driving unit. For a defined voltage in a piezoelectric system, the mechanical inputs change the charge $Q$ on the electrodes (the current through the device). The same happens in a simple sensor, the charge is just converted in a voltage by the devices capacitance.

The charge is calculated with Eqs. (2) and (4), which define the electrical displacement $D$. The charge $Q$ is then given by:

$Q=\int_{A_{\mathrm{E}}} D_{3} \mathrm{~d} A_{\mathrm{E}}$

where $A_{\mathrm{E}}$ is the electrode area. The stress $T_{1}$ in Eqs. (2) and (4) is the stress resulting from mechanical loads and piezoelectric actuation. The part resulting from piezoelectric expansion or compression is the stress that is induced by resisting this action. Also in the free bender it is non-zero.

For the calculation of the charge $Q$, the positions of the neutral plane are important. The stress $T_{1}$ is calculated again from the strain $\varepsilon_{\text {tot }}$ according to Eq. (31) to obtain only the resisting stress we have to subtract the piezoelectric induced strain. According to the sign conventions a positive strain (elongation) is $S_{1}=$ $-d_{31} E_{3}$ :

$T_{1}=\frac{1}{s_{11}^{\mathrm{p}}}\left(\varepsilon_{0}(z+\gamma)+\frac{s_{11}^{\mathrm{s}}}{I} M_{\mathrm{b}}(x)\left(z-z_{0}\right)+d_{31} E_{3}\right)$.

The electric displacement $D$, and therefore the charge $Q$, has to be calculated for the upper and lower layer separately since different electric fields and thicknesses are possible and the stress differs as well. Another point that shows the need for a separate consideration is the $z$-component in $T_{1}$. Although an equation for $D$ with $z$-dependency is possible, Eq. (47) shows that this is impossible for the charge $Q$. The charge $Q$ is technically on the electrodes and can therefore not depend on the coordinate $z$, which is oriented perpendicular to them.

This apparent contradiction can be solved with a thought experiment: the piezoelectric layers are considered to be a stack of infinitesimal small layers. Each layer contributes to the entire piezo layer with a charge according to the stress in the layer. Now it also contributes with its infinitesimal cross-section to the total cross-section. This fact makes it obvious that the average stress is the effective stress. It follows:

$$
\begin{aligned}
& T_{1}^{\mathrm{u}}=\frac{1}{h_{\mathrm{pu}}} \int_{h_{\mathrm{pl}}+h_{\mathrm{s}}}^{h_{\mathrm{pl}}+h_{\mathrm{s}}+h_{\mathrm{pu}}} \frac{1}{s_{11}^{\mathrm{p}}}\left(\varepsilon_{\mathrm{tot}}+d_{31} E_{3}^{\mathrm{u}}\right) \mathrm{d} z, \\
& T_{1}^{\mathrm{l}}=\frac{1}{h_{\mathrm{pl}}} \int_{0}^{h_{\mathrm{pl}}} \frac{1}{s_{11}^{\mathrm{p}}}\left(\varepsilon_{\mathrm{tot}}-d_{31} E_{3}^{1}\right) \mathrm{d} z,
\end{aligned}
$$

The strain $\varepsilon_{\text {tot }}$ is the superposition of strains resulting from external moments and piezoelectric actuation as in Eq. (31):

$\varepsilon_{\mathrm{tot}}(z, x)=\varepsilon_{0}(z+\gamma)+\frac{s_{11}^{\mathrm{s}}}{I} M_{\mathrm{b}}(x)\left(z-z_{0}\right)$.

In this equation, the great importance of the position of the neutral planes can be seen clearly. An error would stay as an offset in the equation and falsify the results of the following calculations.

These stresses put in Eqs. (2) and (4), the charges $Q_{\mathrm{u}}$ and $Q_{1}$ become according to Eq. (43):

$$
\begin{aligned}
& Q_{\mathrm{u}}=\int_{0}^{w} \int_{0}^{L}\left(-d_{31} T_{1}^{\mathrm{u}}+\epsilon_{33} E_{3}^{\mathrm{u}}\right) \mathrm{d} x \mathrm{~d} y, \\
& Q_{1}=\int_{0}^{w} \int_{0}^{L}\left(d_{31} T_{1}^{1}+\epsilon_{33} E_{3}^{1}\right) \mathrm{d} x \mathrm{~d} y .
\end{aligned}
$$

The common approach for all possible configurations, that is described in chapter 2, cannot be used for the calculation of the charge. Eqs. (47) and (48) cannot be always solved if some parameters are zero. With respect to these difficulties the charge has to be calculated for each case (see Fig. 2) separately.

For the triple-layer bender, the charge will be calculated for upper and lower piezo layers separately. The total charge of the system depends then on the electrical connections (parallel or series connection). Eqs. (47) and (48) for the triple-layer bimorph will result into very large equations that are to long to be stated here. To show the function of the principle, the equations will be solved for the heterogeneous bimorph (monomorph) and the homogeneous bimorph as examples.

To calculate the charge of a symmetric bimorph, we simplify Eqs. (15)-(18), as well as Eqs. (26) and (29) according to the bimorph geometry:

$h_{\mathrm{pu}}=h_{\mathrm{pl}}=h_{\mathrm{p}}, \quad h_{\mathrm{s}}=0$.

The simplification has to be introduced in a early stage of the calculation to avoid problems that would make the calculation fail (like a division by zero) as mentioned before. The charge reduces then to:

$$
\begin{aligned}
Q= & \frac{L w}{s_{11}^{\mathrm{p}}}\left(E_{3}^{\mathrm{u}} s_{11}^{\mathrm{p}} \epsilon_{33}-\frac{d_{31}^{2}\left(E_{3}^{\mathrm{u}}+E_{3}^{\mathrm{l}}\right)}{8}\right) \\
& +\frac{3 d_{31}}{4 h_{\mathrm{p}}^{2}} \int_{0}^{L} M_{\mathrm{b}}(x) \mathrm{d} x
\end{aligned}
$$

The presented bimorph will not have any intermediate electrode $\left(h_{\mathrm{s}}=0\right)$, so the charge will be present at the top- and bottom electrodes. The total electric field $E_{3}$ is identical in both piezoelectric layers and can be replaced by the voltage $V$ according to:

$V=E_{3}^{\mathrm{u}}\left(2 h_{\mathrm{pu}}\right)=E_{3}^{\mathrm{l}}\left(2 h_{\mathrm{pl}}\right)$. 
For the monomorph, the simplification will be as follows:

$h_{\mathrm{pu}}=h_{\mathrm{p}}, \quad h_{\mathrm{pl}}=0, \quad E_{3}^{\mathrm{u}}=E_{3}, \quad E_{3}^{1}=0$

The charge, which is on the electrodes of the remaining piezoelectric layer (upper layer) is reduced to:

$$
\begin{aligned}
Q= & \frac{E_{3} L w\left(K \epsilon_{33}-d_{31}^{2} h_{\mathrm{s}}\left(h_{\mathrm{s}}{ }^{3} s_{11}^{\mathrm{p}}+h_{\mathrm{p}}{ }^{3} s_{11}^{\mathrm{s}}\right)\right)}{K} \\
& +\frac{6 d_{31} h_{\mathrm{s}} s_{11}^{\mathrm{s}} s_{11}^{\mathrm{p}}\left(h_{\mathrm{p}}+h_{\mathrm{s}}\right)}{K} \int_{0}^{L} M_{\mathrm{b}}(x) \mathrm{d} x
\end{aligned}
$$

The parameter $K$ in Eq. (53) is still the $K$ from Eq. (16) that simplifies also according to Eq. (52).

\section{Example calculation for a complex monomorph actuator}

\subsection{Mechanical behavior of example monomorph}

To demonstrate the capabilities of the modeling approach an example was chosen that shows its adaptability to different boundary conditions. The configuration of the bender is given in Fig. 10. The bender consists of two layers, a long support layer of the thickness $h_{\mathrm{s}}$ and a shorter piezoelectric layer on top, with the thickness $h_{\mathrm{p}}$. The total beam is simply supported at both ends and the force $F$ is acting on it at $x=a$. The electric field $E_{3}$ has to induce an internal moment that compensates the influence of the force $F$ at the point $x=a$, so that the deflection is zero at that point.

The bending moment is discontinuous in this case, thus two equations are needed:

$M_{\mathrm{b} 1}=-F\left(1-\frac{a}{L}\right) x \quad 0 \leq x \leq a$

$M_{\mathrm{b} 2}=-F\left(\frac{a}{L}\right)(L-x) \quad a \leq x \leq L$

Also two moments of inertia are needed. While the moment of inertia $I$ from Eq. (29) is also valid for the bender for $b \leq$ $x \leq(L-c)$ under the simplifications of Eq. (52), the moment of inertia left and right is different:

$I_{\mathrm{s}}=\frac{w h_{\mathrm{s}}^{3}}{12} \quad 0 \leq x \leq b \quad$ and $\quad(L-c) \leq x \leq L$

With the two equations for the bending moment (54) and (55) and the two moments of inertia (29) and (56), we ob-

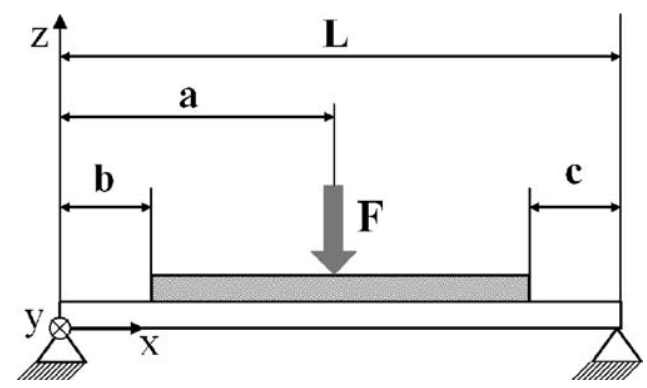

Fig. 10. Configuration of the example bender. tain four different equations for the curvature according to Eq. (32):

$$
\begin{aligned}
& \varepsilon_{1}=\frac{M_{\mathrm{b} 1} s_{11}^{\mathrm{s}}}{I_{\mathrm{s}}} \quad 0 \leq x \leq b \\
& \varepsilon_{2}=\frac{M_{\mathrm{b} 1} s_{11}^{\mathrm{s}}}{I}+\varepsilon_{0} \quad b \leq x \leq a \\
& \varepsilon_{3}=\frac{M_{\mathrm{b} 2} s_{11}^{\mathrm{s}}}{I}+\varepsilon_{0} \quad a \leq x \leq(L-c) \\
& \varepsilon_{4}=\frac{M_{\mathrm{b} 2} s_{11}^{\mathrm{s}}}{I_{\mathrm{s}}} \quad(L-c) \leq x \leq L
\end{aligned}
$$

The benders deflection can now be calculated by double integration according to Eq. (21). The resulting integration constants can be solved with the boundary conditions and transition conditions. These are:

$$
\begin{array}{ll}
\delta_{1}^{\prime \prime}(x=0)=0 & \delta_{1}^{\prime}(x=b)=\delta_{2}^{\prime}(x=b) \\
\delta_{4}^{\prime \prime}(x=L)=0 & \delta_{1}(x=b)=\delta_{2}(x=b) \\
\delta_{3}(x=(L-c))=\delta_{4}(x=(L-c)) & \delta_{2}^{\prime}(x=a)=\delta_{3}^{\prime}(x=a) \\
\delta_{3}^{\prime}(x=(L-c))=\delta_{4}^{\prime}(x=(L-c)) & \delta_{2}(x=a)=\delta_{3}(x=a)
\end{array}
$$

And for the determination of the appropriate electric field $E_{3}$ the deflection at the point $x=a$ is set to zero :

$\delta_{2}(x=a)=0$

The resulting equations are very extended and will not be listed here but with some numerical values the effective curvature can be shown. The numerical values are:

$$
\begin{aligned}
& s_{11}^{\mathrm{s}}=\frac{1}{370 \times 10^{9}} \frac{\mathrm{m}^{2}}{\mathrm{~N}}, \quad s_{11}^{\mathrm{s}}=15 \times 10^{-12} \frac{\mathrm{m}^{2}}{\mathrm{~N}}, \\
& d_{31}=-210 \times 10^{-12} \frac{\mathrm{m}}{\mathrm{V}} \quad h_{\mathrm{p}}=h_{\mathrm{s}}=0.5 \mathrm{~mm}, \quad L=40 \mathrm{~mm}, \\
& w=4 \mathrm{~mm} \quad b=c=5 \mathrm{~mm}, \quad a=20 \mathrm{~mm}, \quad F=0.1 \mathrm{~N}
\end{aligned}
$$

The values are arbitrary but fit a bender of reasonable size. The compliance of the support layer is the one of alumina. The compliance $s_{11}^{\mathrm{p}}$ and the constant $d_{31}$ are taken from the data sheet of an average soft PZT. With these values the electric field can be calculated that is necessary to keep the point $x=a$ at zero deflection. The resulting electric field is $E_{3}=61830.4 \frac{\mathrm{v}}{\mathrm{m}}$, which results in a voltage across the piezoelectric layer of approximately $V=30.92 \mathrm{~V}$.

For printing, the curvature of the total bender, we have to merge the four equations resulting equations. Therefore, we need to introduce the unitstep function $H(x)$.

$H(x-d)=\left\{\begin{array}{l}0 \text { if } x<d \\ 1 \text { if } x \geq d\end{array}\right.$

The total curvature $\delta$ of the beam is then:

$$
\begin{aligned}
\delta(x)= & \delta_{1}(x)(H(x)-H(x-b))+\delta_{2}(x) \\
& (H(x-b)-H(x-a))+\delta_{3}(x) \\
& (H(x-a)-H(x-(L-c)))+\delta_{2}(x)(H(x-L-c))
\end{aligned}
$$




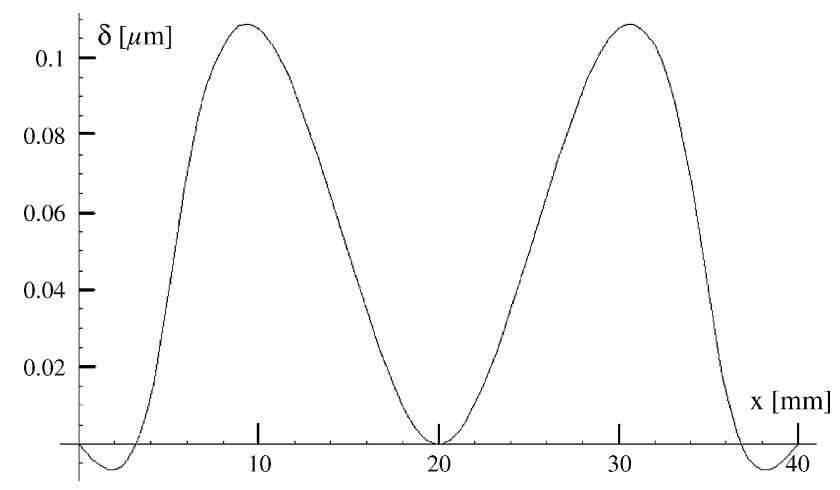

Fig. 11. Bending shape for example bender.

The resulting shape of the bender is depicted in Fig. 11: if the equations for the strain ((57)-(60)) are based on the strain Eq. (31) instead of (32), the strain in any position in the bender can be calculated exactly.

In this example, the strain at the bottom of the support layer (the bottom of the whole system) is calculated and plotted (Fig. 12). The example of strain at the bottom was chosen, because this strain can be measured by strain gauges. Now the model provides the correlation between the force $F$, the electric field $E_{3}$ and the strain $\varepsilon$ at the bottom layer and the deflection $\delta$ at any point. With this knowledge, a system can be established that controls the deflection of the bender by means of applied electric field, with the measurement of the strain. The strain on the bottom layer for two different forces $F=0.1$ and $0.2 \mathrm{~N}$, and their appropriate electric fields $(\delta(x=a)=0)$ is depicted in Fig. 13: with the help of the model the dimensioning, optimization and the ideal placement of the strain gauges for such a system can be done easily.

\subsection{Optimization of example monomorph}

To show the advantage of exact strain modeling the example monomorph will be optimized for strain gauge force measurement.

In a first step, the best position for the strain gauge on the bender has to be chosen. From Fig. 13, it can be seen that the highest strain value on the surface for a certain force is at the point $x=b$. The strain gauge will be placed on the lower surface of the beam $(z=0)$, since the upper surface is uneven due to the transition from passive bender to monomorph (see

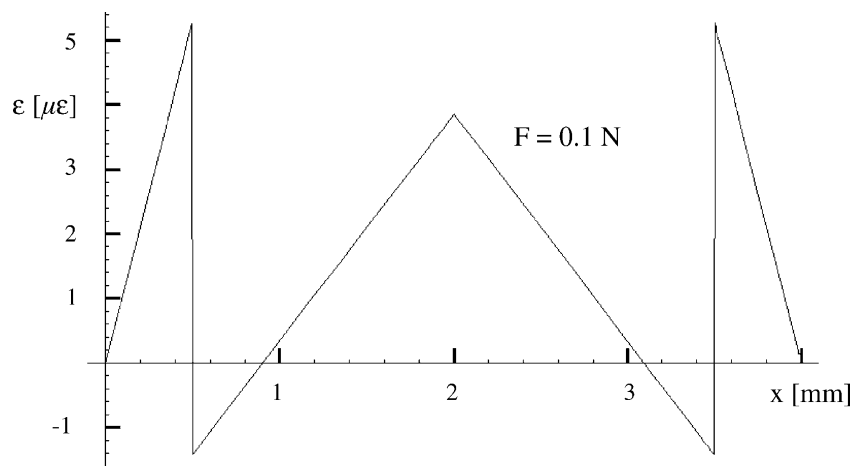

Fig. 12. Strain at the bottom of the example bender.

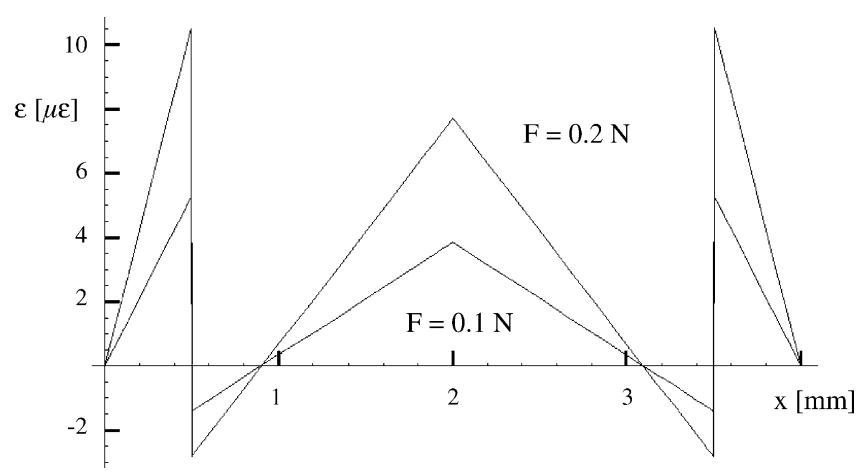

Fig. 13. Strain at the bottom of the example bender for different forces.

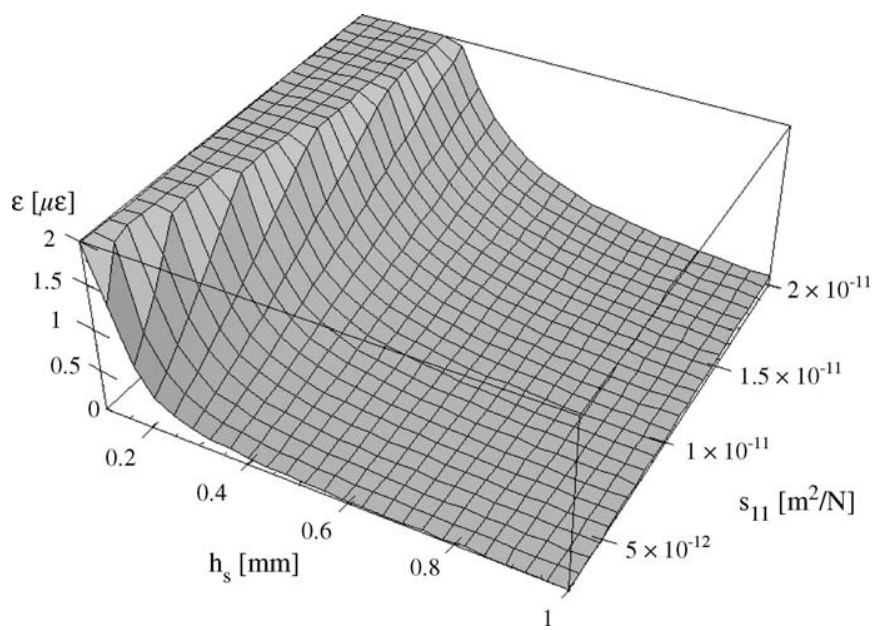

Fig. 14. Strain at $x=b, z=0$ for different thicknesses $h_{\mathrm{s}}$ and compliances $s_{11}^{\mathrm{s}}$.

Fig. 10). Now, we have to maximize $\varepsilon_{\text {tot }}$ :

$\max \left(\varepsilon_{\mathrm{tot}}(x=b, z=0)\right)$

for the maximization of the strain we use Eq. (57) but with the $z$-coordinate (according to Eq. (31)). For the example, we stick to most of the parameters and optimize just for $h_{\mathrm{s}}$ and $s_{11}^{\mathrm{s}}$. This corresponds to a choice of the support layer thickness and material. The resulting strain at this point is shown in Fig. 14. The thickness $h_{\mathrm{s}}$ ranges from $100 \mu \mathrm{m}$ to $1 \mathrm{~mm}$, which fits the unchanged parameters of the actuator. The compliance $s_{11}^{\mathrm{s}}$ ranges from $2.5 \times 10^{-12} \mathrm{~m}^{2} / \mathrm{N}$ (which corresponds to $400 \mathrm{GPa}$ ) to $2 \times 10^{-11} \mathrm{~m}^{2} / \mathrm{N}(50 \mathrm{GPa})$. The diagram (Fig. 14) shows that an optimum does not exist, but for higher compliance $s_{11}^{\mathrm{s}}$ (lower stiffness) and higher thicknesses $h_{\mathrm{s}}$ the strain output increases significantly. (The results in the graph are cut at $2 \mu \varepsilon$, the real values grow ad infinitum.) The real optimum for the application has to be found with the help of other criterions or limitations.

\section{Conclusion}

The modeling approach given has two major advantages compared to conventional models:

(1) The modeling approach is totally independent of mechanical constraints and boundary conditions. Any possible configuration can be modeled as long as the beam theory holds. 
(2) The modeling approach does not only allow the calculation of the curvature (bending angles, deflection, etc.) and electrical properties, it is also capable of modeling the exact strain in any given point of the bender.

The approach has also the advantage, that $x, y$ and $z$ are variable in the equations to the end, so that a plotting of curvature and strain, as in Section 3 is easily possible. The approach is also not just another bender model. The calculation of the actuator contains all necessary parameters, that allows a mathematical optimization. As shown in Section 3.2, the calculation of the strain in the actuator helps finding the best position for the placement of strain gauges as well as the optimization of the actuator geometry to maximize strain gauge output. The effect of the multiple neutral planes, described in Section 2.4 could be evaluated experimentally. The experimental results show the importance of the consideration of the multiple neutral planes for the calculation of the existing strains and stresses.

Since the total approach is based on simple mechanical theories it can be extended easily. In the equations that are stated here for a maximum of three layers, any number of layers can be introduced as long as they still can be handled. For the same reason, the approach can be easily adopted for two-dimensional problems according to the plate theory. The extended model is proven to fit all conditions possible in various applications.

\section{References}

[1] J.G. Smits, S.I. Dalke, T.K. Cooney, The constituent equations of piezoelectric bimorphs, Sens. Actuators A (1991) 28.

[2] J.G. Smits, W.-S. Choi, The constituent equations of piezoelectric heterogeneous bimorphs, IEEE Trans. Ultrason. Ferroelect. Freq. Control 38 (1991) 3.

[3] P. De Lit, J. Agnus, N. Chaillet, The constitutive equations of a piezoelectric duo-bimorph, Proceedings on the fifth IEEE International Symposium on Assembly and Task Planning, Besançon, France, July 10-11, 2003.

[4] M. Weinberg, Working equations for piezoelectric actuators and sensors, J. Microelectromech. Syst. IEEE 8 (1999) 4.

[5] J.-K. Park, W.-K. Moon, Constitutive relations for piezoelectric benders under various boundary conditions, Sens. Actuators A: Phys. 117 (1) (2005) 159-167.
[6] M. Wiesendanger, Squeeze film air bearings using piezoelectric bending elements, Ph.D. thesis, no. 2336, EPFL, 2001.

[7] K. Ruschmeyer, Piezokeramik: Grundlagen Werkstoffe Applikationen,B460, Kontakt \& Studium, Expert Verlag, 1995.

[8] S.P. Timoshenko, D.H. Young, Theory of Structures, second ed., McGrawHill, Auckland [etc.], 1965.

[10] A. Bergander, T. Meader, B. Valencia, J.-M. Breguet, P. Ryser, Integrated sensors for PZT actuators based on thick-film resistors, IEEE MHS, Nagoya, Japan, October 20-22, 2002, 181-186.

\section{Biographies}

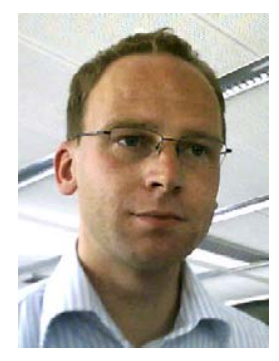

Robert Dunsch graduated from the Dresden University of Technology, Germany, in mechatronics in 2002. In the same year, he joined the "High Precision Robotics group (HPR)" of Jean-Marc Breguet at LSRO, EPFL as a Ph.D. student. His research field is microrobotics, with a focus on modeling of sensor and actuator systems.

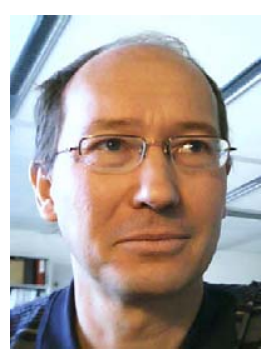

Jean-Marc Breguet graduated at the Engineering High School in Le Locle, Switzerland, in electronic engineering in 1982. In 1989, he graduated at the Swiss Federal Institute of Technology (EPFL) in Lausanne, Switzerland, in micro engineering and in 1998 he obtained is doctorate at EPFL in microrobotics. From 1982 to 1985 and from 1989 to 1993, he worked in industry. Since 1993, he works at EPFL as senior researcher at the "Laboratoire de Systèmes Robotiques (LSRO)" were he created the "High Precision Robotics group (HPR)". HPR is mainly funded by European and national projects in cooperation with industry. His main field of research is microrobotics and its applications in biology and nanotechnology with a special emphasis on piezoelectric actuators and sensors. He gives lectures on robotics, microrobotics, MEMs and precision engineering. In 1998, his work on microrobotics was awarded the "European Grand Prix for Innovation" in Monaco and in 2005, one of his research project was nominated for the "Swiss Technology Award 2005". He is organizer of several conferences ("Microrobotics and Microassembly": SPIE, 1998 to 2000) and workshops (Microrobotics for Biomanipulation: IROS 2003, ...). He is committee member of several conferences and scientific evaluator for the "Natural Sciences and Engineering Research council of Canada (NSERC)". $\mathrm{He}$ is author or co-author of more than 60 publications. Four of them were awarded in conferences. He holds five patents. 